(Aus dem physiologischen Institut der Universität zu Bonn.)

\title{
Ueber den Gehalt des Froschkörpers an Glykogen in den verschiedenen Jahreszelten.
}

Von

J. Athanasiu (Bukarest).

(Mit 1 Textfigur.)

Die Fortschritte, welche im Studium des Glykogens seit seiner Entdeckung gemacht worden sind (Bernard), haben uns gezeigt, welche Rolle diese Substanz im thierischen Körper spielt. - Man kennt den Antheil, welcher dem Glykogen in den Vorgängen des Stoffwechsels zukommt. - Aber wenn man diese Fragen vom Standpunkt der vergleichenden Physiologie betrachtet, so bemerkt man leicht, dass gewisse Verschiedenheiten bei den einzelnen Arten vorhanden sind. - Es gibt im Thierreiche Wesen, die während der kalten Jahreszeit in den unter dem Namen Winterschlaf bekannten Zustand fallen. Die Grösse des Stoffwechsels ist während des Winterschlafes bei ihnen auf ein Minimum reducirt. - Es wäre also interessant zu untersuchen, wie dann das Glykogen sich verhält.

Die Wichtigkeit der Frage war Cl. Be rnard (1859) nicht entgangen, und er hat in der Leber, Muskeln und Lungen des Murmelthieres während des Winterschlafes eine Anhäufung des Glykogens gefunden. - Dieses Glykogen versehwindet aus Muskeln und Lungen sehr schnell wieder, sobald das Thier erwacht.

Nach Ansicht Schiff's (1859) hingegen verminderte sich in der Leber des Murmelthieres während seiner Erstarrung die Menge des Glykogens (thierische Stärke) bedeutend. - Es fehlt sogar vollständig in einem Thiere, das während des Winterschlafes stirbt. Bezüglich der Frösche, behauptet Schiff, dass ihre Leber selbst während des Winters eine grosse Menge Körnchen thierischer Stärke enthält.

Luchsinger (1875) hat in der Leber eines Frosches im November $0,32 \mathrm{~g}$ Glykogen gefunden; Ende December constatirte er 
in einem Falle $0,19 \mathrm{~g}$, in einem andern $0,22 \mathrm{~g}$, jedes Mal in der Leber des Frosches.

Diese Abnahme hat Luchinger zu der Annahme geführt, dass das'Glykogen gegen Ende des Winters vollständig aus dem Körper des Frosches verschwinden muss. Obgleich Luchsinger keinen Beweis als Stütze dieser Annahme gibt, ist sie doch von vielen Physiologen angenommen worden. Man hatte selbst zu einer gewissen Zeit geglaubt, dass das Verschwinden des Glykogens während des Winters nicht auf die Frösche sich beschränke, und wenn man von dem Glykogen der Winterschläfer sprach, so stellte man die Säugethiere mit den Batrachier zusammen, welche den Winter im Zustande des Schlafes verbringen. Aber man hat nicht gezögert, das Studium dieser Frage aufzunehmen, und A eby (1875) hat wie Bernard in der Leber der Murmelthiere während des Winters viel Glykogen gefunden. - Vo it, Kü z (1881), D u b o is (1894) haben ebenfalls gezeigt, dass das Glykogen nicht gänzlich im Körper fehlt und besonders nicht in der Leber der Murmelthiere während ihres Winterschlafes.

Diese Untersuchungen geben uns indess keine Klarheit über das Verhältniss, welches zwischen der Bedeutung des MurmelthierGlykogens während des Winters und während des Sommers besteht. - Die Untersuchungen Dubois' wie die C1. Bernard's an den erwachten Murmelthieren sind kurz nach der Beendigung ihres Schlafes angestellt worden; desshalb haben sie gefunden, dass das Glykogen fast vollständig verschwunden war, was sicherlich dem grossen Verbrauche desselben durch die Organe in diesem Zustande zuzuschreiben ist. Was die andern Säugethiere angeht, so haben Gü r ber (1894) und K i es sel (1896) bewiesen, dass in der Leber eines gut genährten Kaninchens die Menge von Glykogen im Sommer geringer ist $(2,7-4,25 \%)$ als im Winter $(7-11 \%)$.

Auch bei Fröschen sind nur sehr wenige genaue Untersuchungen über die Menge des Glykogens während der verschiedenen Jahreszeiten gemacht worden. - Was den Winter anlangt, finden wir Bestimmungen, welche sich auf die verschiedenen Organe des Frosches (Leber, Muskeln etc.) beziehen, um andere Fragen der Physiologie über Glykogen zu erklären. So untersuchte $K \ddot{u} l z$ (1880), als er suchte, ob der Muskel Glykogen hervorbringen könne, Winterfrösche und fand in ihren Muskeln $0,60-0,66 \%$. - V. Wittich (1883) hat in der Leber des Winterfrosches $3,7-8 \%$ gefunden. 
Aldeh off (1889) hat den Einfluss der Nahrungsentziehung auf das Glykogen studirt und fand in den Versuchen, die er beim Frosche anstellte, ziemlich grosse Mengen Glykogen in den Muskeln und Lebern dieser Thiere selbst nach langen Hungerperioden. - Obgleich durch die Entziehung der Nahrung die Frösche sich in anormalen Zuständen befanden, sehen wir nichtsdestoweniger aus den Zahlen, die Aldehoff angibt, dass das Glykogen, namentlich in der Leber, während der Herbst- und Wintermonate reichlicher sich findet als während des Sommers. - Diese Thatsache scheint auch Dewevre (1892) bemerkt zu haben; denn er behauptet, dass die Leber des Frosches im Anfange des Winters zwei Mal so viel Glykogen enthält als im Sommer. Es nimmt ab in dem Verhältnisse wie der Winter vorschreitet, um am Ende des Wintersehlafes ganz zu versehwinden. - Leider gibt Dewevre keinen Versuch zur Stütze seines Satzes.

Die Thatsachen, welche wir soeben angeführt haben, sind trotz jhrer Wichtigkeit hinsichtlich des Glykogens bei den Winterfröschen, nicht von allen Physiologen in Betracht gezogen worden. Auch Bunge (1891) behauptete, dass gegen den Frühling hin das Glykogen in der Leber des Frosches, der wäbrend des Winters nichts gefressen hat, verschwindet.

In noch jüngerer Zeit hat sich Polimanti (1898) in seiner Arbeit über die Entstehung von Fett durch Phosphor auf die Hypothese Luchsinger's gestützt.

Diese Hypothese ist endgültig vernichtet worden durch P fl ü ge r (1898), der die Entdeckung machte, dass die Frösche beim Ende des Winterschlafes eine grosse Menge Glykogen in ihrem Körper besitzen $(0,992 \%$ bei den Männchen der Rana fusca).

Das Glycogen fehlt auch nicht in den Fröschen, die im Laboratorium aufbewabrt werden, und die keinen Winterschlaf halten $(0,65 \%$ bei den Weibchen der Rana esculenta).

Wir sehen also, dass der Winterschlaf kaum zum Versehwinden des Glykogens im Körper des Frosches beiträgt.

Es handelt sich nun darum, zu erfahren, welche Beziehung zwischen der Menge des Glykogens und dem Winterschlaf besteht. Die Erforschung dieser Frage ist also das Ziel, welches die gegenwärtige Arbeit verfolgt, wobei Herr Professor Pflüger uns mit seinen Rathschlägen beehrt hat. 
Methodik. - Bei allen unsern Versuchen, welche diese Frage betreffen, haben wir so viel als möglich Abweichungen von den natürlichen Lebensbedingungen der Frösche zu vermeiden gesucht, obgleich dies viel weniger bei den Thieren mit wechselnder Temperatur bedenklich ist als bei denen mit constanter Temperatur. - Zur Bestimmung des Glykogens haben wir uns der Methode von Br ückeKülz bedient, mit den Vervollkommnungen, die Pflüger (1898) eingeführt hat. - Die Thiere werden mit Chloroform getödtet und in einer kochenden Lösung von $\mathrm{KOH}(1,7 \%)$ aufgelöst. Das Sieden geht weiter, bis der grösste Theil der weichen Gewebe aufgelöst ist, was gewöhnlich 20-30 Minuten nicht überschreitet. Man lässt die Flüssigkeit abfliessen und fügt dann noch eine kleine Menge Kalilösung hinzu und kocht von Neuem, bis die einzelnen Muskelstückchen, welche noch übrig geblieben waren, aufgelöst sind. In der erkalteten Gesammtmasse der Flüssigkeit fällt man mittelst des Brücke'schen Reagens und Salzsäure die Eiweissstoffe. - Das Ganze wird 24 Stunden ruhig stehen gelassen, und dann filtrirt. Der Niederschlag wird in einer Kalilauge gelöst und wieder durch Salzsäure ausgefällt. Dieser Vorgang wird noch 2-3 Mal wiederholt, bis das Filtrat keine Trübung mit Alkohol mehr zeigt. - Das Glykogen wird mit zwei Volumina Alkohol von $96 \%$ niedergeschlagen, dann nach den Anweisungen von $\mathrm{Külz}$ gereinigt und bei $100^{\circ}$ getrocknet.

Ausgehend von den Versuchen Pflüger's, die im März 1898 gemacht wurden, haben wir das Glykogen der Frösche in den Monaten Juni, Juli, September, October, November und Februar (1899) bestimmt. - Die Ergebnisse unserer Versuche haben wir in der auf S. 565 gegebenen Tabelle zusammengestellt.

Die Prüfung dieser Ziffern zeigt uns einegeringere Menge Glykogen im Körper des Frosches während des Sommers.

Den Gang des Glykogengehaltes beim Frosche während eines Jahres verfolgend, können wir am besten aus folgender Bogenlinie ersehen, in welcher wir auf den Abscissen die Mengen des Glykogens als Ordinaten für die verschiedenen Monate eingetragen haben.

Wir sehen an dieser Bogenlinie, dass die Menge des Froschglykogens ihr Maximum im Herbst und ihr Mimimum im Sommer hat. 
Ueber den Gehalt des Froschkörpers an Glykogen etc.

\begin{tabular}{|c|c|c|c|c|c|c|c|}
\hline $\begin{array}{c}\text { Nummer } \\
\text { der } \\
\text { Ver- } \\
\text { suche }\end{array}$ & Datum & Gattung & Geschlecht & $\mid \begin{array}{c}\text { Anzahl } \\
\text { der } \\
\text { Frösche }\end{array}$ & $\begin{array}{c}\text { Ge- } \\
\text { sammt- } \\
\text { gewicht }\end{array}$ & $\begin{array}{c}\text { Glykogen } \\
\text { Gesammt } \\
\text { menge }\end{array}$ & $\begin{array}{l}\text { In \% der } \\
\text { Lebenden- } \\
\text { Substanz }\end{array}$ \\
\hline $\begin{array}{c}1 \\
2 \\
3 \\
4 \\
5 \\
6 \\
7 \\
8 \\
9 \\
10 \\
11 \\
\left.12^{1}\right) \\
13 \\
14 \\
15 \\
16 \\
17 \\
18\end{array}$ & 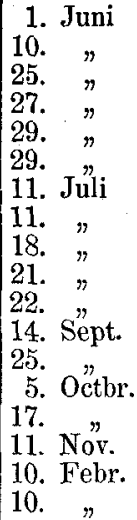 & $\begin{array}{c}\text { Esculent. } \\
\text { Fusca } \\
\text { Esculent. } \\
\quad " \\
" \\
\text { Fusca } \\
" \\
" \\
" \\
\text { Esculent. } \\
" \\
" \\
\text { Fusca } \\
" \\
"\end{array}$ & $\begin{array}{c}\text { Männchen } \\
" \\
" \\
" \\
\text { Weibchen } \\
\text { Männchen } \\
\text { Weibchen } \\
\text { Männchen } \\
\text { Weibchen } \\
\text { Männchen } \\
\text { Weibchen } \\
" \\
" \\
\text { Männchen } \\
\text { Weibchen }\end{array}$ & $\begin{array}{r}6 \\
4 \\
15 \\
10 \\
8 \\
22 \\
2 \\
4 \\
5 \\
16 \\
7 \\
12 \\
31 \\
10 \\
10 \\
12 \\
6 \\
12\end{array}$ & $\begin{array}{r}231 \\
144 \\
302 \\
333 \\
121 \\
360 \\
72 \\
159 \\
143 \\
444 \\
175 \\
350 \\
729 \\
223 \\
428 \\
573 \\
279 \\
698\end{array}$ & $\begin{array}{c}0,909 \\
0,523 \\
0,910 \\
1,330 \\
0,138 \\
0,620 \\
0,338 \\
0,780 \\
0,750 \\
1,280 \\
0,64 \\
1,64 \\
10,43 \\
2,92 \\
5,61 \\
5,20 \\
3,00 \\
7,33\end{array}$ & $\begin{array}{l}0,392 \\
0,362 \\
0,301 \\
0,400 \\
0,113 \\
0,117 \\
0,467 \\
0,489 \\
0,531 \\
0,28 \\
0,368 \\
0,470 \\
1,43 \\
1,31 \\
1,31 \\
0,907 \\
1,07 \\
1,05\end{array}$ \\
\hline
\end{tabular}

Aber wir haben in der Tabelle und in der Bogenlinie die Ergebnisse zusammengestellt, die bei den männlichen und weiblichen Fröschen der Rana esculenta gefunden worden sind.

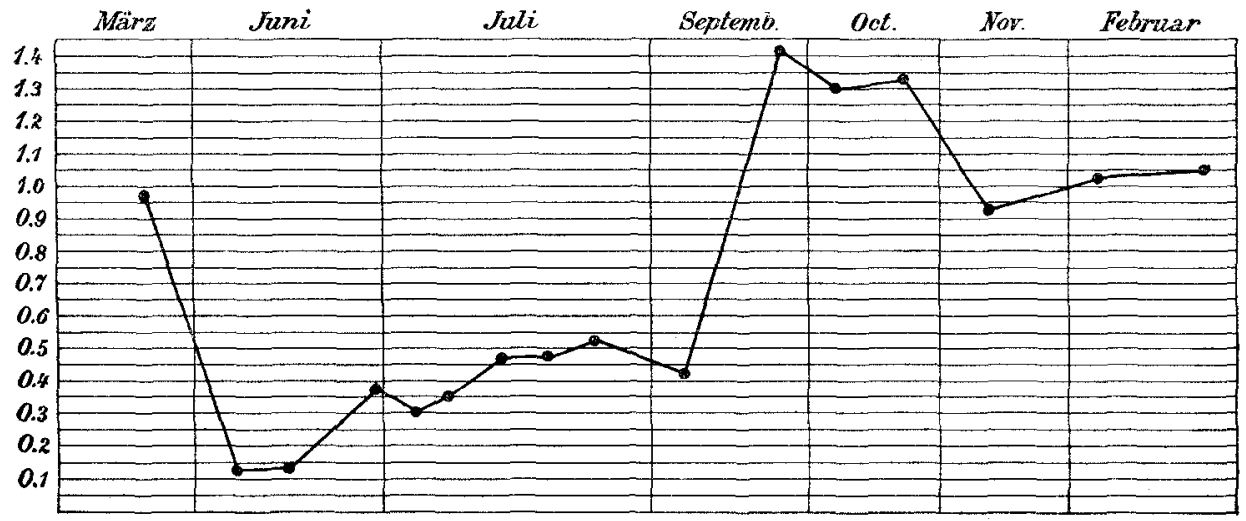

Fig. 1.

Es wäre nicht ohne Bedeutung, die Vertheilung des Glykogens in den verschiedenen Körpertheilen während des Verlaufes eines Jahres zu kennen. - Bezüglich des Frühjahrs haben wir folgenden Versuch gemacht: 28. März. - 30 weibliche Frösche (Rana fusca),

1) Dieser Versuch ist in Bukarest gemacht worden. 
im Gewichte von zusammen $705 \mathrm{~g}$, wurden mit Chloroform getödtet. - Dann bestimmte man nach einander das Glykogen in den Lebern, den Muskeln, den Häuten, im Centralnervensystem und in den Eiern:

\begin{tabular}{|c|c|c|c|c|c|c|c|}
\hline 25 & Leber & $=$ & 2,27 & g & Glykogen & $=$ & $8,73 \%$ \\
\hline 225 & Muskel & $=$ & 2,25 & $\eta$ & $\eta$ & $=$ & $1,00 \%$ \\
\hline 175 & Häute & $=$ & kein & & " & $=$ & - \\
\hline 2,3 & Centralnervensystem & $=$ & 0,001 & $n$ & $"$ & $=$ & $0,07 \%$ \\
\hline 50 , & Eier & $=$ & 0,55 & $n$ & $"$ & $=$ & $1,1 \%$ \\
\hline
\end{tabular}

Wir sehen also, dass im Frühling wieder die Leber die gröste Menge Glykogen enthält.

Was die übrigen Zeiten des Jahres betrifft, so entnehmen wir die Zahlen von Glykogen den Controlfröschen, die uns zu den Versuchen mit Phosphor gedient haben ${ }^{1}$ ). - Wir bemerken hierzu, dass bei diesen Versuchen die Thiere einige Zeit gehungert hatten. Ferner ist das Glykogen in den Verdauungsflüssigkeiten nach der Methode von Salkowsky-Austin (1897) bestimmt worden. Nichtsdestoweniger sind die gefundenen Zahlen nicht sehr verschieden von denen, die sich bei den andern Versuchen ergeben haben.

\begin{tabular}{|c|c|c|c|c|c|c|c|c|c|c|}
\hline \multirow{2}{*}{ 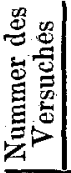 } & \multirow[b]{2}{*}{ 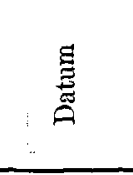 } & \multirow[b]{2}{*}{ 点 } & \multirow{2}{*}{ 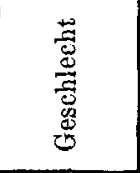 } & \multirow{2}{*}{ 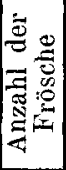 } & \multirow{2}{*}{ 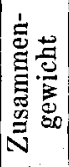 } & \multicolumn{3}{|c|}{ Glykogen in } & \multirow[b]{2}{*}{ 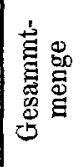 } & \multirow[b]{2}{*}{ 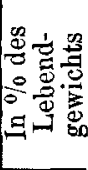 } \\
\hline & & & & & & 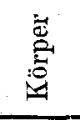 & 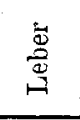 & 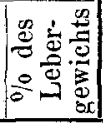 & & \\
\hline $\begin{array}{r}8 \\
9 \\
10 \\
11 \\
12\end{array}$ & $\begin{array}{l}\text { 17. Juli } \\
25 . \text {. } \\
\text { 27. Sept. } \\
\text { 7. Octbr. } \\
\text { 11. Nov. }\end{array}$ & $\begin{array}{l}\text { Fusca } \\
\text { Esculent. } \\
\quad " \\
\text { Fusca }\end{array}$ & $\begin{array}{c}\text { Männchen } \\
" \\
" \\
" \\
"\end{array}$ & $\begin{array}{l}15 \\
20 \\
10 \\
12 \\
12\end{array}$ & $\begin{array}{l}533 \\
640 \\
256 \\
449 \\
624\end{array}$ & $\begin{array}{l}1,289 \\
1,62 \\
0,87 \\
1,88 \\
1,13\end{array}$ & $\begin{array}{l}0,443 \\
0,87 \\
1,24 \\
1,56 \\
1,88\end{array}$ & $\begin{array}{l}2,77 \\
4,35 \\
8,26 \\
8,21 \\
7,52\end{array}$ & $\begin{array}{l}1,732 \\
2,49 \\
2,11 \\
3,44 \\
3,01\end{array}$ & $\begin{array}{l}0,324 \\
0,390 \\
0,820 \\
0,760 \\
0,482\end{array}$ \\
\hline
\end{tabular}

Betrachten wir jetzt die Glykogenmenge in der Leber, so sehen wir, dass sie dieselbe Schwankung erleidet wie beim ganzen Körper (weniger, wenn die äussere Temperatur hoch ist, mehr wenn sie niedrig ist).

Es ergibt sich aus diesen Versuchen, dass die Menge des im Körper des Frosches vorhandenen Glykogens im Allgemeinen umgekehrt proportional ist der umgebenden Temperatur. - Nachdem dies zugegeben ist, wollen wir sehen, welche Beziehung zwischen diesen beiden Klassen von Erscheinungen besteht. - Suchen wir 
zuerst die Ursache für die Verminderung des Glykogens im Sommer $\mathrm{zu}$ finden. Der Einfluss der umgebenden Temperatur ist der erste Factor, den wir kennen müssen. - $\mathrm{Zu}$ diesem $\mathrm{Zwecke}$ nehmen wir unsere Zuflucht zum Experimentiren.

Schiffer (1881) hatte gefunden, dass die Menge des Glykogens in den Muskeln der Frösche zunimmt, wenn man sie an einen Ort mit $30^{\circ}$ bringt. - So findet er in den Muskeln des Frosches im Winter ein Durchschnittsverhältniss von $0,77 \%$ Glykogen. Nach einer Erhitzung von 3 Stunden steigt die Menge auf $1,09 \%$, und nach 24 Stunden auf $1,33 \%$. - Diese Thatsache darf zwar überraschen, wenn man sich erinnert, dass der Stoffwechsel beim Frosche direct proportional ist der umgebenden Temperatur. - Es wäre aber denkbar, dass die Glykogenzunahme in den Muskeln von der Stärke ihrer Oxydationen abhängig ist. - Dieses Glykogen käme wahrscheinlich aus der Leber dank der Thätigkeit des Blutkreislaufes. Wenn dem so ist, so muss das Glykogen in der Leber abnehmen, besonders wenn seine Bildung die Verluste nicht aufwiegt. - Dieses scheint der Fall zu sein, und die Versuche Moiszeik's (1888) zeigen, dass man das Glykogen in der Leber des Frosches zum Verschwinden bringen kann, wenn man sie zwei Wochen lang in einer Temperatur von $25^{\circ}$ belässt. - Ebenso hat Schulte-Overberg (1894) gefunden, dass beim Kaninchen, wenn es in einer Temperatur von 33-38 gehalten wird, die Menge des Leberglycogens von $4-5 \mathrm{~g} \mathrm{\%}$ auf $0,002 \mathrm{~g}$ bis $0,3 \mathrm{~g} \%$ zurückgeht.

Wir haben auch gesucht zu finden, welchen Einfluss die experimentelle Erhitzung auf das Gesammtglykogen des Frosches ausübt.

7. October. - 33 weibliche Frösche (Rana esculenta), die alle am selben Orte gefunden wurden, haben wir in zwei Gruppen getheilt: a) 17 Frösche im Gesammtgewicht von 447,5 g wurden 10 Tage hindurch in einer Temperatur von $22-24^{\circ}$ gehalten; b) 16 Frösche im Gewichte von $420 \mathrm{~g}$ wurden dieselbe Zeit hindurch bei $14^{\circ}$ gehalten.

$\begin{array}{ccc} & \begin{array}{c}\text { Gesammtmenge } \\ \text { des Glykogens }\end{array} & \begin{array}{c}\% \text { der } \\ \text { lebenden Substanz }\end{array} \\ \text { Gruppe a . . . . . . . } 3,74 \mathrm{~g} & 0,837 \\ \text { Gruppe b . . . . . } 5,65 \% & 1,34\end{array}$

Also die experimentelle Erhitzung hat ebenso wie die natürliche Wärme des Sommerseine Verminderung der gesammten Glykogenmenge zur Folge. 
Suchen wir jetzt die Erklärung für diese Erscheinung zu finden. - Zuerst müssen wir uns erinnern, dass es für das Glykogen wie für jede Ernährungssubstanz eine bestimmte Bilanz zwischen der Bildung des Glykogens und seinem Verbrauche gibt. - Daraus folgt, dass seine Menge durch grössere Bildung oder durch geringeren Verbrauch zunehmen kann und umgekehrt. - Da dies nicht bezweifelt werden kann, so handelt es sich nur darum, zu erfahren, welcher von diesen beiden Factoren in Frage kommt, damit das Glykogen unter dem Einflusse der Hitze abnehme.

Da eine grössere Hitze, wie besonders durch $\mathrm{H}$. Schulz bewiesen wurde, die Oxydationsprocesse und die Arbeit der Organe bei den Fröschen steigert, so werden auch mehr Kohlehydrate verbraucht und den Organen hierdurch Fnergie zugeführt.

Also durch zu starken Verbrauch von Glykogen vermindert die Hitze dessen Menge. - Es ist wohl möglich, dass durch die Temperatur die Glykogenbildung auch verstärkt wird; aber da der Verlust grösser ist, so ergibt sich als Schlussresultat eine Verminderung in der Gesammtmasse.

Dieser grosse Verbrauch von Glykogen während des Sommers entspricht sicherlich zwei Bedürfnissen: erstens um den Organfunctionen zu genügen, welche während dieser Jahreszeit am stärksten sind; zweitens um die Ansammlung von Fett zu erleichtern, welches das Thier als werthvollen Vorrath für den Winter aufbewahrt. - Dieser Fettvorrath ist es, welcher vor Allem während der kalten Jahreszeit benützt wird, und man kann dies aus dem Umstande schliessen, dass die Fettkörper im Frühlinge fast verschwunden sind.

Die Schlussfolgerung aus dem Gesagten ist, dass das Glykogen, welches man am Schlusse des Winterschlafes im Körper der Frösche findet, nur ein Rest des Vorrathsstoffs sein kann, der dem geringen Verbrauche durch den Organismus während des Winters zuzuschreiben ist.

\section{Literat ar.}

1859. C1. Bernard, De la matière Glycogène comme condition de developpement de certains tissus chez le foetus avant l'apparition de la fonction Glycogenique du foie. Compt. rend. Acad. Sciences t. 48 p. 673-684. Paris. 
1859. M. Schiff, De la nature des granulations qui remplissent les cellules hépatiques: Amidon animal. Compt. rend. de l'Acad. des Sciences t. 48 p. $880-884$.

1872. E. Pflüger, Ueber die Diffussion des Sauerstoffes, den Ort und die Gesetze der Oxydationsprocesse im thierischen Organismus. Arch. f. die gesammte Physiol. Bd. 6 . S. 43.

1875. B. Luchsinger, Beiträge zur Physiologie und Pathologie des Glykogens. Dissert. Zürich.

1875. C. Aeby, Archiv für experimentelle Pathologie und Pharmak. S. 184.

1880. E. Külz, Bildet der Muskel selbständig Glykogen? Arch. f. d. gesammte Physiologie Bd. 24 S. 64.

1880. J. Schiffer, Ueber den Einfluss der Temperatur auf den Glykogengehalt der Froschmuskeln. Centralbl. f. d. med. Wochensebr. Nr. 18.

1883. W. Wittich, Physiologie der Aufsaugung, Lymphbildung und Assimilation. Hermann's Handbuch der Physiologie Bd. 5 II. Th. S. 367.

1888. O. Moszeik, Mikroskopische Untersuchungen über den Glykogenansatz in der Froschleber. Arch. f. Physiologie Bd. 42 S. 556.

1889. G. Aldehoff, Ueber den Einfluss der Carenz auf den Glykogenbestand von Muskel und Leber. Zeitschr. f. Biologie Bd. 25 S. 137.

1891. G. Bunge, Cours de chimie biologique et pathologique. p. 337.

1892. Dewevre, Note sur la fonction glycogénique chez la grenouille d'hiver Comp. rend. hebd. de la Soc. d. Biologie p. 19-21. Paris.

1894. G. Schulte-Overberg, Ueber die Einwirkung hoher Aussentemperatur auf den Glykogenbestand der Leber. Dissertation. Würzburg.

1894. A. Gürber, Die Glykogenbildung in der Kaninchenleber zu verschiedener Jahreszeit. Sitzungsbericht der Würzburg. phys.-med. Gesellschaft.

1896. O. Kissel, Die Glykogenbildung in der Kaninchenleber zu verschiedener Jahreszeit. Verhdl. d. phys.-med. Gesellsch. zu Würzburg Bd. 30 S. 77-82.

1897. A. E. Austin, Ueber die quantitativen Bestimmungen des Glykogens in der Leber. Arch. f. path. Anat. u. Physiol. Bd. 150 S. 185-196.

1897. O. Polimanti, Ueber die Bildung von Fett im Organismus nach Phosphorvergiftung. Arch. f. d. gesammte Physiol. Bd. 70 S. 349-365.

1898. E. Pflüger, Beiträge zur Physiologie der Fettbildung, des Glykogens und der Phosphorvergiftung. (Unter Mitwirkung von Herrn J. A th a nasiu.) Arch. f. die gesammte Physiol. Bd. 71 S. 318-332. 